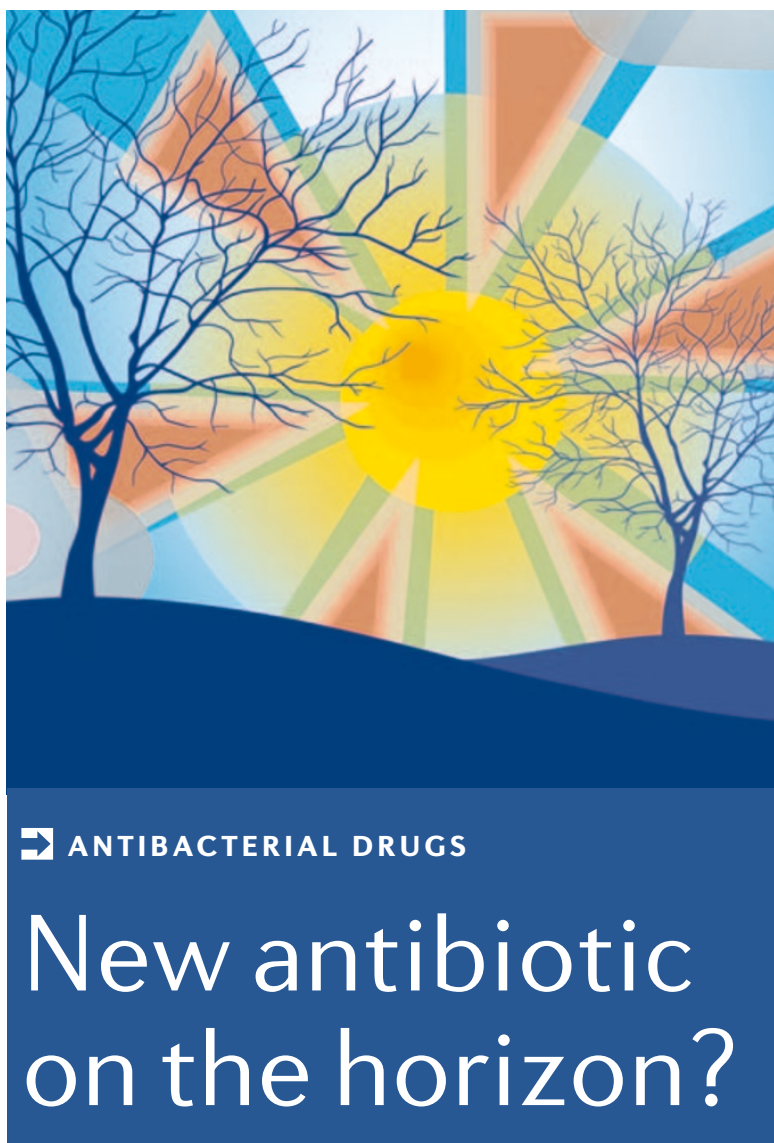

A return to investigating natural products as a source of antimicrobials could hold the key to tackling multidrug resistant bacteria. Writing in Nature, Wang and colleagues describe the isolation and characterization of platensimycin, a small molecule that represents a new class of antibiotic and which has broad, potent activity against Gram-positive pathogens.

A pathway yet to be fully exploited for antibiotic drug discovery is fatty acid biosynthesis. A key enzyme involved in this pathway is

first-in-class
antibacterial
drug.

the $\beta$-ketoacyl-acyl carrier protein synthase FabF. To increase the chance of identifying a compound that selectively targets FabF, the authors engineered the Gram-positive pathogen Staphylococcus aureus to express antisense RNA against FabF. This renders the bacteria more sensitive to inhibitors of this enzyme, and so comparison of growth inhibition of the wild-type strain with that of the antisense strain enables the identification of genuine FabF-selective inhibitors present in the fermentation samples. This screen identified a potent antibiotic, platensimycin, which could otherwise have been overlooked in a conventional screening assay.

Platensimycin was found to have broad-spectrum activity against Gram-positive bacteria in in vitro assays and was able to eradicate systemic $S$. aureus infection in mice. Importantly, platensimycin shows no cross-resistance with other classes of antibiotics, and is active against bacterial infections that are resistant to commercially available drugs, such as methicillin-resistant S. aureus and vancomycin-resistant enterococci.

To verify that the mode of action of platensimycin was the selective targeting of FabF, the authors used a radioactive platensimycin derivative. Initially, the binding affinities of the derivative for FabF were much lower than anticipated, which led the authors to propose that platensimycin was targeting the transiently formed (and so difficult to detect) acyl-FabF intermediate. Indeed, preparation of a stable acyl-enzyme complex to mimic the transient form increased the binding signal and confirmed the authors' hypothesis that platensimycin only binds to the acyl intermediate.

The transient nature of the acyl-FabF complex also made attempts to obtain a crystal structure of platensimycin bound to its target challenging. However, by substituting the cysteine residue in the active site of the enzyme with glutamine, the side chain of which is thought to mimic a bound fatty acid, the authors produced a stable FabF variant-platensimycin complex that was amenable to crystallographic analysis. From this they were able to determine that platensimycin targets the malonate-binding site of FabF, thereby blocking fatty acid biosynthesis, and that the formation of the acyl-FabF intermediate opens up the active site of FabF, a conformational change that is essential for platensimycin binding.

In recent decades, there has been a dearth of new classes of antibiotic. Platensimycin is currently the most potent inhibitor of FabF, as well as being the only inhibitor of this enzyme that has broad-spectrum activity. Fatty acid biosynthesis is so far an under-targeted pathway, and so it has considerable potential for drug development. On the basis of these data, and with structural information in hand, platensimycin looks to be a promising first step towards a new first-in-class antibacterial drug.

Samantha Barton
RESEARCH HIGHLIGHTS ADVISORS

ERIK DE CLERCQ

Katholieke Universiteit Leuven, Belgium RODERICK FLOWER

William Harvey Research Institute, QMW,

London, UK

YOSHIJI FUJITA

Clinical Proteome Center, Tokyo Medical University
F. PETER GUENGERICH

Vanderbilt University Nashville, TN, USA FRANZ HEFTI

Rinat Neuroscience Corporation, Palo Alto, CA, USA

JOAN HELLER BROWN

University of California San Diego,
Un, CA, USA
MADS KROGSGAARD THOMSEN

Novo Nordisk, Bagsvaerd, Denmark HUGO KUBINY

University of Heidelberg, Germany ROBERT LANGER Massachusetts Institute of Technology Cambridge, MA, USA JULIO LICINIO University of California Los Angeles, CA, USA
CHRISTOPHER LIPINSKI

Pfizer Global Research and Development, Groton, CT, USA TOMI SAWYER

Ariad Pharmaceuticals, Cambridge, MA, USA JANET WOODCOCK

Food \& Drug Administration, Rockville, MD, USA 


\section{A new treatment for liver fibrosis?}

Chronic liver injury in response to alcohol or hepatitis virus $B$ or $C$ is associated with liver fibrosis, and its endstage, cirrhosis, is a major public health problem. However, at present, there are no effective antifibrotic drugs approved for human use. In a recent paper in Nature Medicine, Teixeira-Clerc and colleagues now show that use of the cannabinoid CB1 receptor antagonist rimonabant could be a new therapeutic strategy for this condition.

The authors recently found that during the course of chronic hepatitis C, daily cannabis use is an independent predictor of fibrosis progression. They therefore sought to determine if $\mathrm{CB} 1$ receptors might be involved in liver fibrosis.

Firstly, using immuno-blotting and immunohistocytochemistry, TeixeiraClerc et al. showed that $\mathrm{CB} 1$ receptor expression was upregulated in human cirrhotic liver samples, predominantly in hepatic myofibroblasts, the fibrogenic cells of the liver. They then used a murine model of acute liver injury to demonstrate that $\mathrm{CB} 1$ receptors are involved in the induction of two fibrogenic markers: the profibrogenic cytokine transforming growth factor (TGF)- $\beta 1$ and smooth muscle $\alpha$-actin. Administration of rimonabant reduced expression of both markers, and there was a similar reduction in fibrogenic marker expression in CB1 knockout mice, with or without rimonabant treatment.

To further assess the role of $\mathrm{CB} 1$ receptors in chronic liver injury, three mouse models were used: chronic $\mathrm{CCl}_{4}$ intoxication, chronic thioacetamide intoxication and bile duct ligation. Rimonabant lowered the fibrogenic response, independently of the agent used to induce liver injury, as shown by a decrease in fibrosis area, reduced hepatic expression of TGF- $\beta 1$, and decreased number of liver fibrogenic cells. Similar reduction of fibrogenesis was observed in CB1 knock-out mice.

The authors then focused on how antagonism of CB1 receptors might reduce the accumulation of hepatic myofibroblasts. Hepatic myofibroblasts from $\mathrm{CB} 1$ receptor knockout mice displayed increased apoptosis, whilst rimonabant was able to inhibit their proliferation. They also investigated the phosphatidylinositol 3-kinase-Akt and extracellular-regulated kinase (ERK) pathways, which are necessary for the growth and survival of hepatic myofibroblasts. Cells isolated from CB1 knockout mice showed decreased phosphorylation of ERK and Akt, as did rimonabant-treated wild-type hepatic myofibroblasts, suggesting that CB1 receptors on hepatic myofibroblasts may either be activated by an endogenous ligand or be constitutively active.

Overall, these results clarify the profibrogenic role of the $\mathrm{CB} 1$ receptor. And although yet to be tested for liver fibrosis in clinical trials, this study opens the possibility that rimonabant, which has been recommended to receive marketing authorization for the treatment of obesity by the European Medicines Agency, might also have potential in the treatment of liver fibrosis.

Charlotte Harrison

ORIGINAL RESEARCH PAPER Teixeira-Clerc, T. et al. CB1 cannabinoid receptor antagonism: a new strategy for the treatment of liver fibrosis. Nature Med. 12, 671-676(2006)

FURTHER READING Lotersztajn, S. et al. Hepati fibrosis: molecular mechanisms and drug targets. Annu. Rev. Pharmacol. Toxicol 45, 605-628 (2005) Kunos, G. et al. Cannabinoids hurt, heal in cirrhosis. Nature Med. 12, 608-609 (2006)

\section{Homing in on the target of antidepressants}

The mechanism of action of most commonly used antidepressants is poorly defined and a better understanding of the effects of these drugs on the brain could lead to new therapeutic approaches.

\section{A potential link between} neurogenesis and antidepressant treatment announced several years ago generated excitement in the field, but the mechanisms underlying this connection have remained elusive. Now a new study by Enikolopov and colleagues narrows down the target of at least one class of antidepressants to a specific population of early progenitor cells.
Neurons are continuously generated in the dentate gyrus region of the hippocampus throughout life. In animal models, this activity increases in response to various types of antidepressant therapy, including selective serotonin reuptake inhibitors (SSRIs) such as fluoxetine, and potentially contributes to their therapeutic effects. However, the precise stage of the multi-step process of neurogenesis at which SSRIs actually intervene is unknown. A clearer understanding of the progenitor cell type targeted by these drugs could enhance understanding of the pathogenesis of depression and the development of improved antidepressants.

Quantification and characterization of different progenitor cell types by immunofluorescence is difficult, owing to the widespread, cytoplasmic expression of typical marker proteins such as nestin, combined with dense packing of the cells within the dentate gyrus. The authors therefore generated mice in which a fluorescent signal was localized specifically to the nucleus of these cells, producing a labelling pattern that was easier to quantify. In combination with staining for various proteins that are expressed at different stages of neurogenesis, the authors defined six distinct sequential stages of neuronal development and investigated the effects of chronic fluoxetine treatment on the proliferative activity of cells at each stage. Using this approach, the authors showed that the cells targeted by fluoxetine to increase neurogenesis are 'amplifying neural progenitors' - the cells generated in the second 'stage' of neurogenesis as defined in this study. 


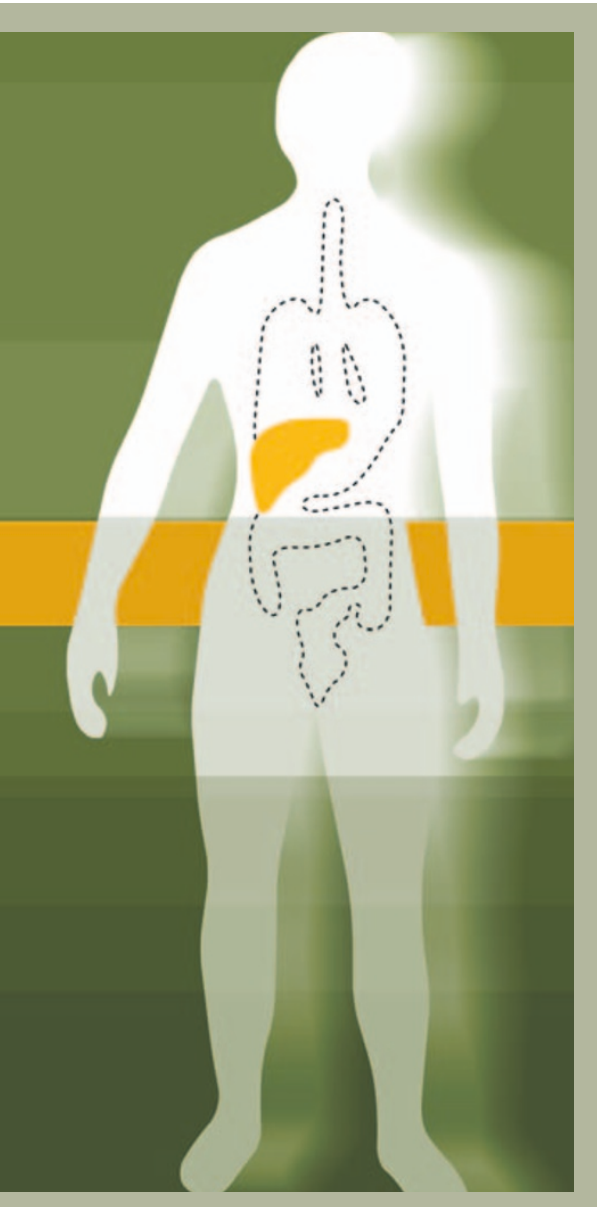

It will be vital to determine whether these cells are a common target for other types of antidepressant, and it remains to be confirmed whether these effects will translate to the human condition. Nevertheless, this study sheds light on the cells targeted by SSRIs in the brain and should direct further studies to define the precise molecular targets of these drugs. Furthermore, this model allows different early progenitor types to be more clearly defined, which could contribute to a better understanding of both hippocampal neurogenesis and the pathological effects of depression.

Katherine Whalley

ORIGINAL RESEARCH PAPER Encinas, J. M. et al. Fluoxetine targets early progenitor cells in the adult brain. Proc. Natl Acad. Sci. USA 103. 8233-8238 (2006)

FURTHER READING Duman, R. S. et al. Regulation of adult neurogenesis by antidepressant treatment. Neuropsychopharmacology 25, 836-844 (2001)| Santarelli, L. et al. Requirement of hippocampal neurogenesis for the behavioural effects of antidepressants. Science 301, 805-809 (2003)

\section{Unpicking the pathogenesis of sepsis}

Severe sepsis, which results from the body's response to infection, remains a leading cause of death and disability, despite decades of intensive research and billions of dollars of investment in the development of potential therapies. Aird and colleagues, reporting in the Journal of Experimental Medicine, now show that vascular endothelial growth factor (VEGF) seems to have a key role in the pathogenesis of sepsis, and could represent a promising new target for therapeutic intervention.

A major challenge in drug development for sepsis is that the complex interplay between mediators of the inflammatory and coagulation pathways that are involved in the disorder is poorly understood. Recently, however, studies have indicated an association between severe sepsis and elevated levels of VEGF, a key promoter of endothelial permeability and proliferation that also seems to have pro-inflammatory and procoagulant effects. Aird and colleagues therefore set out to test the idea that VEGF has a pathogenic role in sepsis.

First, the authors assayed plasma levels of VEGF and the related placental growth factor (PIGF) in mouse and human models of infection, and found that sepsis is associated with increased expression and circulating levels of VEGF and PIGF. Peak levels occurred later than those of early-response cytokines such as tumour-necrosis factor- $\alpha$ (TNF $\alpha$ ), interleukin-1 (IL-1) and IL-6.

VEGF mediates its effects through binding to two transmembrane receptor tyrosine kinases, VEGFR1 (also known as Flt-1) and VEGFR2 (also known as Flk-1), whereas PIGF binds to VEGFR1. A naturally occurring soluble form of VEGFR1 is also known to bind to VEGF and PIGF, and thereby block their interaction with cell-surface receptors. So to investigate the potential for therapeutic intervention, and the roles of these various proteins, Aird et al. assessed the effects of several anti-VEGF strategies on sepsis pathogenesis.

In the first strategy, adenovirus-mediated overexpression of a soluble form of VEGFR1 in mouse models of sepsis attenuated the rise in free VEGF and PIGF levels, and blocked the adverse effects of endotoxaemia on cardiac function, vascular permeability and mortality. A second strategy, involving pre-treatment with anti-VEGFR1 or anti-VEGFR2 antibodies, revealed that anti-VEGFR2 antibodies, but not anti-VEGFR1 antibodies, reduced mortality, which suggests that it is VEGF, and not PIGF, that is a crucial mediator of the sepsis phenotype. In addition, assessing the expression levels of several inflammatory and coagulation mediators indicated that VEGF sensitizes endothelial cells to TNF.

Finally, to investigate whether VEGF inhibition might be used therapeutically, mice were treated with soluble VEGFR1 after the onset of sepsis, which resulted in a marked improvement in cardiac physiology and survival. It therefore seems that further studies to determine both the diagnostic/prognostic value of VEGF and the therapeutic potential of antiVEGF strategies in sepsis are warranted. Such studies might be initiated relatively rapidly, as several anti-VEGF agents have been approved for cancer treatment: the anti-VEGF antibody bevacizumab, and the small-molecule VEGFR kinase inhibitors sunitinib and sorafenib.

Peter Kirkpatrick

ORIGINAL RESEARCH PAPER Yano, K. et al. Vascular endothelial growth factor is an important determinant of sepsis morbidity and mortality. J. Exp. Med. 203, 1447-1458 (2006)

FURTHER READING Buras, J. A., Holzmann, B. \& Sitkovsky, M.

Animal models of sepsis: setting the stage. Nature Rev. Drug Discov. 4, 854-865 (2005)

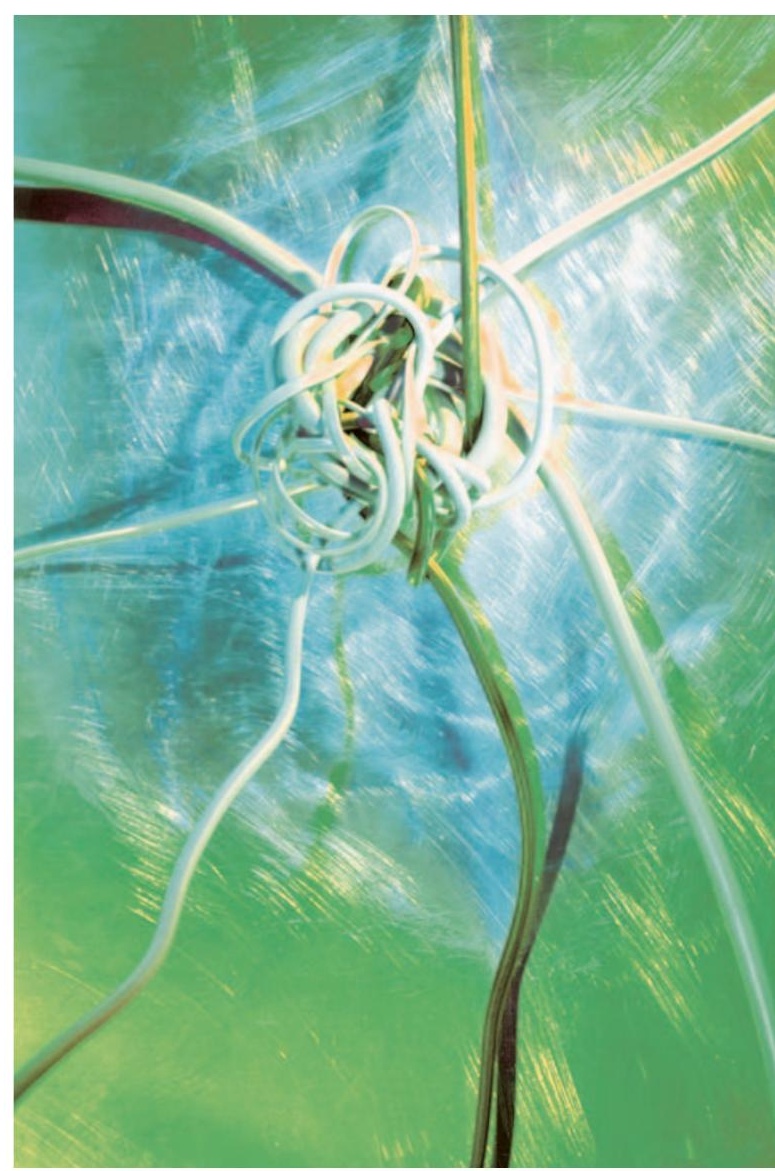


